\title{
LES FIGURES DE CONSTRUCTION DANS LES TEXTES PERSUASIFS D'APRĖS L'EXEMPLE DES ANNONCES MATRIMONIALES
}

\begin{abstract}
Otulak-Komenda Monika, Les figures de construction dans les textes persuasifs d'après l'exemple des annonces matrimoniales [The figures of construction in the persuasive texts on the example of signales advertisements], Studia Romanica Posnaniensia, Adam Mickiewicz University Press, Poznań, vol. XXXVII/2: 2010, pp. 29-38. ISBN 978-83-232-2189-0. ISSN 0137-2475. DOI 10.2478/v10123-0100012-4.

A sender of a personal ad, who usually does not have any theoretical knowledge of rhetoric, intuitively uses simple schemes in the text which are based on the syntactic modifications of zero level. The schemes, which are discussed in this article, do not introduce any changes on the semantic level, as a result, they do not cause ambiguity and they do not make the text's reception more difficult. On the one hand, making such schemes is easy, by and large, it does not demand the knowledge of theoretical basis of rhetoric. On the other hand, the schemes have an influence on rhythmicality and dynamic of the text. Consequently, they make the reception of the text easier and, in addition, the text becomes more attractive for a virtual recipient.
\end{abstract}

L'annonce matrimoniale constitue un type particulier de texte usuel qui est produit en vue de faciliter ou de permettre de créer des liens interpersonnels. Son but pour l'émetteur consiste à présenter une sorte d'offre pour trouver un futur compagnon. Cette offre est transmise par un médium (de préférence la presse écrite, souvent spécialisée ou Internet, où on trouve un nombre considérable de sites et de portails qui ne publient que des annonces, en particulier des annonces matrimoniales) où les destinataires virtuels peuvent la voir et y répondre, si le message leur correspond. Le texte de l'annonce permet d'entrer en contact avec celui qui pourrait nous donner le bonheur de la vie en couple. Le message devrait être formulé de telle manière qu'il attire l'attention des destinataires. C'est pourquoi les textes des annonces sont fondés sur des procédés différents, autant psychologiques que linguistiques. Une annonce matrimoniale ${ }^{1}$ est un texte persuasif qui constitue une sorte d'autopublicité et en tant que

\footnotetext{
${ }^{1}$ Pour notre analyse nous avons choisi 334 annonces publiées sur Internet sur les sites suivants : www.union-conseil.com et www.vivastreet.fr. Pour les mettre en ordre nous avons signé chaque annonce, d'abord par la lettre signifiant le site ( $\mathrm{U}$ et $\mathrm{V})$, ensuite le sexe $(\mathrm{F}$ et $\mathrm{H})$, finalement le numéro. De plus, nous avons également décidé de garder la graphie et l'orthographe des textes des annonces, bien que ceux-ci soient pleins de fautes de grammaire, d'orthographe et de ponctuation. De telle
} 
tel utilise les techniques propres à ce genre de textes. Pour attirer l'attention du destinataire et pour le convaincre d'accepter, le message publicitaire est riche en moyens agissant sur l'intellect ou sur les émotions. Les auteurs des textes publicitaires n'hésitent donc pas à aller aux sources de la persuasion, c'est-à-dire à la rhétorique et aux techniques qui lui sont propres. Dans les annonces nous trouvons une gamme assez vaste de figures rhétoriques, et dans la plupart des cas les figures dont la création est la plus simple, souvent inconsciente et n'exigeant pas de savoir théorique. Ces figures dynamisent les textes, en leur donnant des traits propres et originaux. Leur usage transmet beaucoup d'informations sur l'émetteur, sur son caractère, sur sa formation ou sur son intuition linguistique et psychologique. Il faut se rendre compte que l'usage de certaines tactiques autoprésentationnelles et de certaines figures rhétoriques ne résulte pas d'habitude du savoir théorique. Les émeteurs n'utilisent pas de manuels de rhétorique ou d'autoprésentation, mais ils créent les annonces automatiquement, conformément à leur intuition.

Dans cette analyse, en nous appuyant sur le classement des figures provenant de la tradition rhétorique de Pierre Fontanier (1977), reprise ensuite par Karl Cogard (2001), nous nous focalisons sur les figures rhétoriques de construction.

Les figures de construction font partie des figures de mots dont le trait caractéristique est que « les mots sont pris dans un sens propre quelconque, c'est-à-dire dans une de leurs significations habituelles ou ordinaires, primitives ou non » (Fontanier, 1977, p. 66). Dans le cas des figures de construction les modifications d'un degré zéro consistent à la « manière dont les mots sont combinés et disposés entre eux dans la phrase » (Fontanier, 1977, p. 66). Elles organisent le niveau syntaxique des textes en n'introduisant pas de changements au niveau du sens.

Parmi les figures de construction nous distinguons : les figures de répétition et les figures de disposition. Les premières consistent à répéter les éléments du texte en séquences différentes, tandis que les deuxièmes sont fondées sur la disposition particulière des éléments en profitant des particularités de la syntaxe, ainsi que du sémantisme des mots. Comme figures de répétition nous allons présenter : l'anadiplose, l'anaphore, l'épanaphore, l'épiphore, la polyptote et le polysyndète, et comme figures de disposition: l'anacoluthe, l'antimétabole, l'asyndète, l'hyperbate, l'oxymore et la syllepse.

Afin de définir les figures nous nous appuyons sur les descriptions proposées par Cogard K. (2001), Korolko M. (1998), Dubois J., et d'autres (Le Groupe $\mu$ ) (1970).

\section{ANADIPLOSE}

C'est la reprise, au début de la phrase suivante ou du segment suivant, d'un mot ou de la séquence de quelques mots qui apparaissent à la fin de la phrase précédente,

manière, les fautes qui apparaissent dans les textes cités, ne sont pas les nôtres, mais sont celles des annonciateurs. 
même si cette reprise n'est ni absolument contiguë ni rigoureusement parfaite. Cette figure permet de souligner un élément du discours - un ou quelques mots. La répétition après une pause syntaxique met ce mot en relief et témoigne de son importance. Contrairement aux autres figures de répétition qui sont très fréquentes, dans les annonces, nous n'avons trouvé que quelques exemples d'anadiplose. Dans l'un d'eux, c'est le mot amour qui est exposé :

Elle ne conçoit pas la vie sans voyages, sans sorties, sans culture et surtout sans AMOUR. Cet amour, elle veut le vivre avec un $\mathrm{H}$ ayant charme et charisme (UF, 124).

\section{ANAPHORE}

Cette figure consiste en la répétition au moins une fois d'un même terme dans un segment du discours. Elle sert à marquer, à exposer une idée. Nous pouvons l'appeler la reine des figures fondées sur la syntaxe, vu sa popularité. Celle-ci résulte tout d'abord de sa facilité de création, ainsi que de sa capacité à attirer l'attention du destinataire. L'anaphore et ses variantes comme épanaphore et épiphore renforcent la segmentation du discours en unités rythmiques et le rythme de sa part facilite la réception du texte. Comme figure rhétorique, on considère l'anaphore fidèle qui consiste en la répétition exacte d'un mot (cf. Cogard, 2001) et non pas au remplacement du mot par son synonyme ou par un pronom (anaphore infidèle).

Dans les textes des annonces nous avons trouvé des exemples de tous les types d'anaphores. D'abord, nous allons présenter quelques anaphores nominales qui consistent en la répétition d'un substantif :

j'ai le sens des responsabilités et le sens de l'humour (UH, 166).

sans projet illusoire, mais projet durable ( $\mathrm{VH}, 327)$.

Une partie considérable des anaphores nominales répète les substantifs désignant le sexe, p.ex. :

Homme d'esprit, homme de cœur (UH,220).

il a la délicatesse et la sensibilité d'un $\mathbf{H}$ de coeur, le charme et la présentation d'un $\mathbf{H}$ de goût (UH, 182).

« Femme active, femme pressée », femme élégante, femme actuelle (UF, 35).

Nous avons aussi trouvé quelques exemples de l'anaphore fondée sur la répétition du mot vie, p.ex. :

A, jusqu'à présent, privilégié sa vie professionnelle. Souhaite maintenant réussir sa vie de couple (UH, 190).

Dans les textes, nous avons plusieurs exemples d'anaphores verbales, d'abord avec le verbe aimer :

Aime la nature, la mer, le soleil, la musique, le sport... enfin, elle aime la vie, l'humour, l'amour (UF, 57). 
J'aime lire, j'apprécie la nature, la marche en forêt, j'aime le cinéma, la peinture, mais seule je n'ai rien envie de faire (UF, 81).

Ensuite, avec le verbe être :

Div., je ne suis pas du tout faite pour la solitude. 38 ans, je suis une jolie F raffinée ou décontractée, c'est selon les circonstances. Cadre (Infimière), la vie m'intéresse. Tolérante, loyale, je suis aussi sensuelle et j'ai de l'humour. Je suis prête à partager mes rêves, mes projets, mes frissons avec un $\mathrm{H}$ charmant, solide, élégant et curieux (UF, 43).

Dans cet exemple cette anaphore pourrait être plutôt considérée en tant qu'erreur stylistique - elle n'assume pas la fonction persuasive ni n'embellit le discours. Elle montre la pauvreté lexicale et stylistique du langage de son auteur, son rôle persuasif est de cette manière renversé.

Et le verbe vivre :

Pense que ses petits revenus vous permettront de vivre plus aisément et surtout de vivre à deux dans la tendresse (UF, 102).

On a trouvé ainsi quelques anaphores adjectivales :

mijoter de bons petits plats, faire des petits voyages (UF, 117).

libre et celibataire [sic !], d'origine maghrébine,sérieux,instruit,desire [sic !] lier une relation durable en vue mariage [sic !] avec une femme francaise, libre et celibataire [sic !] (VH, 298). Femme de bon caracterere [sic !] et bon ton (VH, 299).

Je suis tendre mais la vie n'a pas été tendre avec moi (UF, 12).

et les anaphores adverbiales :

Je l'imagine romantique, très doux, très tendre, joyeux aussi, 27/36 ans, cél., attaché aux vraies valeurs (UF, 2).

j'ai beaucoup voyagé et beaucoup travaillé (UF, 100).

un charme fou, plutôt tendre, plutôt drôle (UH, 185).

Très actif (grands voyages) et très musicien (guitariste), très gourmet aussi (UH, 149).

Elle a l'air si jeune, si douce, si jolie avec ses yeux couleur myosotis qu'on a peine à croire qu'elle assume d'aussi larges responsabilités (UF, 33).

Dans les annonces, nous avons aussi trouvé plusieurs exemples d'anaphores adjectivales, fondées sur la répétition des adjectifs possessifs, p.ex. :

Je ne te connais pas, mais tu me manques, ta douceur, tes rires, ton intelligence, le son de ta voix et ta main dans la mienne (UH, 160).

Il souhaite partager ses rires, ses frissons et sa vie de tous les jours avec F épanouie, sincère, souriante, aimant rire et aimer (UH, 212).

avec qui partager mes rires, ma vie et mes gâteaux (UH, 138).

On l'apprécie pour sa personnalité, sa maturité, son sérieux mais aussi pour sa tendresse et sa gentillesse (UH, 167).

Son entourage l'apprécie pour sa loyauté, sa franchise, son sérieux, sa sincérité et son romantisme qui le rend particulièrement touchant $(\mathrm{UH}, 168)$.

Parfois, les émeteurs lient ensemble quelques types d'anaphores ou les mélangent avec les autres figures de répétition. Dans l'annonce : 
je recherche l'homme de ma vie l'homme qui saura m'aprecier [sic !] a [sic !] ma juste valeure [sic !] et qui prendra soin de moi et je prendrai aussi soin de lui (VF, 244).

la valeur répétitive de l'anaphore nominale homme est renforcée par le polysyndète du pronom relatif qui et l'antimétabole avec la polyptote qui renversent la distribution des rôles dans l'expression prendre soin.

\section{ÉPANAPHORE}

Cette figure est fondée sur la répétition d'une même expression en tête de phrase ou de strophe dans un segment du discours. Parfois, l'épanaphore est appelée anaphore, par extension. Constituant le type d'anaphore, elle est le plus souvent fondée sur le commencement de quelques propositions par la même construction sujet-verbe: on répète le même sujet et le même verbe.

Dans quelques cas, c'est le verbe aimer :

j'aime la campagne, j'aime cajoler, j'aime la cuisine (VF, 238).

J'aime la nature, les étoiles, les enfants, les balades, nager, lire, recevoir, cuisiner. J'aime les JF mignonnes, douces, discrètes et faciles à vivre et j'aime surtout celle qui sera mienne (UH, 144)

Ou le verbe être :

C'est un JH attiré par les voyages, la nature, les restos, le bricolage, les sorties... C'est un grand sentimental qui s'engagera totalement dans un couple (UH, 136).

Et le verbe penser :

Je pense à un petit déjeuner d'amoureux, à des matins clairs pleins de promesses, je pense aux rire complices d'un $\mathrm{H}$ et d'une F. Je pense à cette solitude dont je ne veux plus (UF, 18).

Une autre partie du discours, comme l'adjectif ou l'interjection peut aussi constituer le noyau de l'épanaphore :

Seule dans ce mande [sic !].. seule avec ces chagrins.. seule dans une [sic !] pays etrangeres [sic !] (VF, 261).

\section{ÉPIPHORE}

Cette figure constitue une autre variété d'anaphore. C'est la répétition d'un même mot ou d'une même expression à la fin d'une phrase ou d'un segment du discours. Elle permet d'exposer le sens lié au mot-clef du discours. L'épiphore n'est pas aussi populaire que d'autres figures de répétition.

Dans les textes des annonces, nous avons trouvé un seul exemple de cette figure :

Et même si vous n'aimez pas tout cela, moi j'aimerai ce que vous voudrez là ou vous le voudrez (VH, 332). 


\section{POLYPTOTE}

La polyptote consiste en la répétition de différentes formes flexionnelles d'un même mot. Cette figure concerne particulièrement les formes verbales ou nominales avec le changement du cas (dans les langues ayant la flexion nominale), du nombre. $\mathrm{Vu}$ l'absence de la flexion casuelle dans la langue française, elle est fondée sur la répétition de formes verbales, variées selon la personne, le nombre, le temps, la voix, etc. ainsi que les formes adjectivales, variées selon le nombre et le genre :

il a pourtant toujours cru au couple et continue à y croire (UH, 186).

J'aime le soleil, le ski, le cinéma, faire la fête et dialoguer. Mais, plus que tout, ce que j'aimerais, c'est te rencontrer et te bercer dans ma tendresse (UH, 128).

Elle n'a pas besoin de se faire remarquer pour qu'on la remarque (UF, 20).

Il souhaite partager ses rires, ses frissons et sa vie de tous les jours avec $\mathrm{F}$ épanouie, sincère, souriante, aimant rire et aimer (UH, 212).

Et même si vous n'aimez pas tout cela, moi j'aimerai ce que vous voudrez là ou vous le voudrez (VH, 332).

Je ne sais pas si c'est vrai, mais je voudrais que ce soit vrai pour vous (UH, 176).

Vous lui apporterez votre finesse, votre humour, votre tendresse, Bertrand vous apportera sa solidité, sa tendresse, une aisance et une sécurité affective et matérielle (UH, 187).

Une femme sérieuse cherche un homme sérieux (VF, 267).

\section{POLYSYNDÈTE}

Cette figure consiste à répéter et à multiplier dans un fragment du texte des termes de liaison, comme p.ex. les conjonctions ou les pronoms relatifs. La répétition de la marque de liaison est le plus souvent fondée sur la conjonction la plus populaire et la plus fréquente : la conjonction et:

Carine : douce et fine, chaleureuse et tendre, boucles blondes et regard bleu (UF, 22).

Vous possédez classe et élégance physique et morale, goût de la communication et vous aimez les voyages (UF, 80).

Chaleureuse et accueillante, elle répand autour d'elle un climat apaisant et harmonieux. Jolie dame blonde, féminine et élégante (UF, 112).

On me dit souriante et pleine de vie, câline et facile à vivre (UF, 113).

Une F remarquable et remarquée. Une belle personnalité, pleine d'humour et de chaleur mais aussi de tact et de savoir-vivre. Virginie est d'une vive curiosité intellectuelle, tout l'intéresse (ciné, voyages, littérature, peinture, sorties). Son futur compagnon sera un $\mathrm{H}$ doux et fort, fou et sage, intelligent et tolérant, (UF, 65);

Voici un exemple avec la conjonction mais :

cherche un copain ou ami sans préjugés bon milieu mais pas snob cultivé mais pas pédant (VF, 260).

La répétition peut aussi concerner d'autres conjonctions et des marques de liaison comme les pronoms relatifs : 
Si vous avez comme elle des goûts simples, si vous êtes franc, attentionné, la cinquantaine (UF, 69).

je cherche une femme marocaine, qui cherche un homme qui a vraiment envie d'aimer et de choyer.j'ai mes qualités et mes défauts, mais, je sais que je veux une femme qui a du coeur et qui a envie. (VH, 311);

Céline n'aime ni la tiédeur, ni les demi-mesures (UF, 48).

Le répertoire des conjonctions répétées est assez restreint : on utilise les conjonctions les plus simples et les plus populaires qui n'exigent pas une syntaxe spécifique ou des formes verbales concrètes (comme le subjonctif). Même si on utilise la construction hypothètique avec $s i$, on choisit la construction temporelle la plus simple : si + présent - présent.

\section{ANACOLUTHE}

C'est la rupture de construction syntaxique. Dans la graphie, elle est souvent marqué par le point de suspension. Néanmoins, on ne peut pas considérer chaque utilisation de ce signe de ponctuation comme une anacoluthe car dans la plupart des cas il ne joue que le rôle d'un ornement de la graphie monotone. Comme anacoluthes, nous pouvons considérer des exemples cités ci-dessus :

Si la vie ne fût [sic !] pas simple..., aujourd'hui, je veux rire, sourire et aimer !! (VF, 250). je suis inscrite depuis... ba (VF, 271).

Le but est de se faire plaisir mutuellement, resto, ciné, et si affinités,... (VH, 316).

Et ça sera le début du bonheur... n'hésitez pas, qui que vous soyez, à me contacter comme ça on se voit ds la journée si je suis là et... VOILA! (VH, 333).

Belle réussite professionnelle et peu de temps à penser à sa vie privée. Aujourd'hui, c'est chose faite... (UH, 156).

\section{ANTIMÉTABOLE}

L'antimétabole est une figure qui consiste à l'inversion de la distribution des rôles dans le discours. Elle oppose deux actions, exprimées souvent par le même verbe avec le changement de la voix : l'agent de la première action devient le patient de la deuxième et la subit. Cette figure est très efficace dans le cas des annonces matrimoniales par le fait qu'elle permet de montrer la symétrie de la couple. De cela résulte la présence des verbes de sentiment, comme aimer ou des verbes décrivant la vie en couple, comme être, prendre soin :

je veux juste un homme qui sera à moi, moi je serai à lui (VF, 238).

qui prendra soin de moi et je prendrai aussi soin de lui (VF, 244).

Elle n'a pas besoin de se faire remarquer pour qu'on la remarque (UF, 20).

il veut retrouver le plaisir d'aimer et d'être aimé (UH, 173).

La disposition des rôles peut être aussi fondée sur des verbes antonymes :

elle a trop d'amour à donner et à recevoir (UF, 29). 


\section{ASYNDÈTE}

Cette figure fait l'économie des termes induisant une articulation logique entre les groupes syntaxiques (les propositions, les phrases). C'est une construction dépourvue des mots de liaison, qui permet de marquer le dynamisme du discours, ainsi que d'indiquer la force et le pouvoir de l'émetteur. Cependant, il est presque impossible de trouver des exemples de textes totalement dépourvus des marques de liaison, comme les conjonctions. Dans les textes, on n'observe que des courts passages exprimant cette attitude - elle n'apparaît pas comme la conséquence de choix conscients, mais par hasard, comme le résultat du manque de respect envers la grammaire et l'orthographe. Voilà pourquoi, nous nous limitons à citer quelques exemples d'asyndètes formulées inconsciemment nous semble-t-il :

photo sur demande, aucun probleme [sic !].. origine gabonaise (VF, 251).

femme fr origine israel douce timide ch homme plus 45ans imperatif (ou s'abstenir) ARABE seul region parisienne plutot autoritaire aimant fesser corriger obeissance et calins assures < si mail DETAILLE avec age ville origine desirs maries bye pas la peine commentaires je les efface sans lire annonce SERIEUSE (VF, 256) (j'ai repris la version originale de cette annonce, avec tous les erreurs).

\section{HYPERBATE}

C'est le prolongement inattendu par un mot d'un ensemble syntaxiquement complet. On ajoute à la phrase qui paraissait terminée une épithète, un complément ou une proposition. En effet, cela introduit une sorte de rupture de la construction typique, et en même temps une sorte de développement. Néanmoins, ce n'est pas une seule définition de ce terme, parce que, anciennement, il était utilisé pour nommer une figure qui consiste en l'inversion de l'ordre des mots dans un syntagme (cf. Korolko, 1998, p. 110). Dans les annonces matrimoniales, l'hyperbate apparaît comme un ajout d'informations, soit sur l'émetteur, soit sur ses exigences envers le destinataire. Ces informations peuvent concerner l'âge ou le fait d'avoir des enfants. Puisque cette figure ne joue pas un rôle persuasif considérable, nous ne citerons qu'un exemple, montrant cette attitude :

il veut vivre un amour tendre et fou avec une JF féminine, naturelle, chaleureuse et tendre, aimant nature et enfants, il n'en a pas et acceptera les vôtres avec joie, la quarantaine (UH 175).

\section{OXYMORE}

Cette figure de style associe des termes sémantiquement incompatibles, contradictoires où l'un paraît exclure logiquement l'autre. Il attire l'attention du destinataire, en suscitant souvent un effet de choc sémantique et stylistique. Cette figure ne consti- 
tue pas un élément important dans les annonces. Nous pouvons supposer que c'est à cause du choc qu'elle provoque d'habitude. Une telle émotion pourrait constituer la raison de rejeter une annonce qui comporte l'oxymore. Voilà pourquoi nous n'avons trouvé qu'un seul exemple de l'usage de cette figure, pas très choquant en fait :

un foyer intime et ouvert sur le monde extérieur (UF, 16).

\section{SYLLEPSE}

C'est le jeu sur un signifiant qui a simultanément deux signifiés différents. Le mot peut apparaître quelques fois dans un texte, à chaque fois en ayant une signification différente. La syllepse est une figure fondée sur l'ambiguïté des mots et suscite l'effet d'un jeu de mot, d'un jeu sur le signifiant et le signifié. Voici des exemples de cette figure intéressante :

Un cadre et un style de vie raffinés, une vie prof. Intense (UF, 61).

Dans cette annonce ce sont les significations du mot vie qui sont la base de la figure : la vie comprise comme «manière de vivre, aspect particulier que prennent ces activités et ces événements selon l'individu (style de vie) » et comme « le type d'activité qui s'exerce dans certaines conditions, certains domaines (vie professionnelle) » (Le Petit Robert, 2001, p. 2672).

Dans l'annonce :

Il aime aller au fond des choses, à l'essentiel et maintenant pour lui l'essentiel est de vous rencontrer et de vous aimer (UH, 159)

On joue sur le mot essentiel, compris comme « qui est ce qu'il est par son essence, absoluet comme » « qui est le plus important» (Le Petit Robert, 2001, p. 918).

Les figures de construction organisent le niveau syntaxique et le rythme des textes et leur fréquence est considérable.

Premièrement, comme nous l'avons déjà mentionné, leur création n'exige pas un effort ni un savoir particulier et résulte souvent de l'intuition. Voilà pourquoi elles apparaissent avec une telle fréquence dans les textes créés par les « amateurs ». Cela concerne particulièrement les figures fondées sur la répétition pure des mots sans jouer avec leur sémantisme. Comme le remarque Fontanier, aucune figure n'est une invention des rhéteurs ou des grammairiens : « elles nous viennent de la Nature même, comme la parole» (Fonanier, 1977, p. 67). Les rhéteurs pour leur part n'en ont élaboré que les noms et se sont contentés ensuite de décrire et classer les figures existantes. D'autre côté, comme la construction des figures de disposition est plus difficile et pas tellement automatique, elles apparaissent plus rarement.

Deuxièmement, les figures de construction permettent de dynamiser les textes en introduisant un rythme particulier. Bien que les textes des annonces soient destinés 
à être lus à voix basse, le rythme facilite la réception et rend le texte plus attrayant et plus remarquable.

Troisièmement, leur usage ne provoque pas d'ambiguïtés et de malentendus au niveau de la réception et de la compréhension. Chaque action persuasive, afin d'être complète, exige la présence et la participation de l'émetteur et du destinataire. La persuasion a en effet un caractère interpersonnel et son succès ne dépend pas seulement de l'émetteur, mais de son savoir sur les préférences et le savoir du destinataire, de ses besoins. Mirosław Korolko (1998) appelle cette attitude comme le degré de connaissance du langage du destinataire. La réception de ces figures n'exige aucun savoir particulier, contrairement au cas des tropes. Il n'est pas nécessaire de les connaître au niveau théorique -, de les identifier pour comprendre la totalité du texte.

Un autre élément remarquable, c'est le répertoire des mots sur lesquels sont fondés les figures présentées. En ce qui concerne les substantifs, ce sont les substantifs désignant les sexes (homme, femme) et d'autres substantifs liés à l'amour, à la liaison, comme amour, vie. Au niveau des verbes, on remarque la fréquence des verbes du même champ sémantique : aimer, vivre. Parmi les autres parties du discours, la présence des adjectifs possessifs est très visible. C'est parce que dans les annonces on souligne différents aspects de la liaison : on parle de soi-même, de son physique, de ses préférences, on décrit le destinataire virtuel et on présente la vie commune.

Est-ce qu'une annonce simple et spontanée attire plus l'attention du destinataire virtuel qu'un texte au style original et élaboré? La réponse permettrait de décrire l'effet perlocutoire de ce type des texte et de vérifier la force des tactiques persuasives. Néanmoins, nous laissons cette question ouverte comme existent tant ceux qui préfèrent des textes simples, que ceux qui peuvent se faire tenter par des mots tendres et recherchés.

\section{BIBLIOGRAPHIE}

Cogard, K. (2001). Introduction à la stylistique. Paris : Flammarion, Coll. Champs Université.

Dubois, J. (1970). Rhétorique générale. Paris : Librarie Larousse.

Fontanier, P. (1977). Les figures du discours. Paris : Flammarion.

Korolko, M. (1998). Sztuka retoryki. Warszawa : Wiedza Powszechna.

Le Petit Robert (2001). Paris : Dictionnaires le Robert.

Pierrot, A.H. (1993). Stylistique de la prose. Paris : Bellin.

(s.d.). Récupéré sur http://www.union-conseil.com/UnionConseil.htm

(s.d.). Récupéré sur http://rencontres-homme.vivastreet.fr/annonces-rencontre-homme

(s.d.). Récupéré sur http://rencontres-femme.vivastreet.fr/annonces-rencontre-femme 